\title{
Path optimizing for 3D printing
}

\author{
ZENG Feng \\ School of Computer, Jia Ying University, Meizhou 514015, China \\ 28251245@qq.com
}

Keywords: 3D printing, path optimization, rapid prototyping, 3D CAD model

\begin{abstract}
In the 3D printing process, the 3D CAD model is built in CAD system and sliced into a series of 2D slices. The 2D cross section data contains a lot of unnecessary idle running. If transform the original data into processing path code directly, the processing efficiency will be significantly reduced. A path optimization algorithm is developed in this paper. The minimum spanning tree strategy is utilized in this algorithm. The starting point and the ending point of every single loop are resorted. The shortest processing path of $2 \mathrm{D}$ cross section is acquired by this algorithm. The case study results show that the efficiency of whole 3D printing is increased.
\end{abstract}

\section{Introduction}

The 3D printing technology which belongs to Rapid Prototyping is based on computer numerical control, computer graphics, materials science and technology, computer aided design, digital manufacturing, and laser technology ${ }^{[1-4]}$. In the 3D printing process, the 3D CAD model was built in CAD system and then sliced into a series of $2 \mathrm{D}$ slices ${ }^{[5-6]}$. After that, the $2 \mathrm{D}$ cross section data was obtained and transformed into NC codes, and then the 3D product was accumulated step-by-step and layer-by-layer in 3D space ${ }^{[7]}$. 3D printing process has the following steps, and which is shown in Fig. 1 and Fig. $2^{[8-10]}$.

Step 1. The 3D model of product is built by CAD system.

Step 2. Slicing software slices the 3-D CAD model with certain thickness after analysis and selection of forming direction.

Step 3. Under the control of computer, the 2D cross section of current layer is processed by the laser beam or the micro drop nozzle.

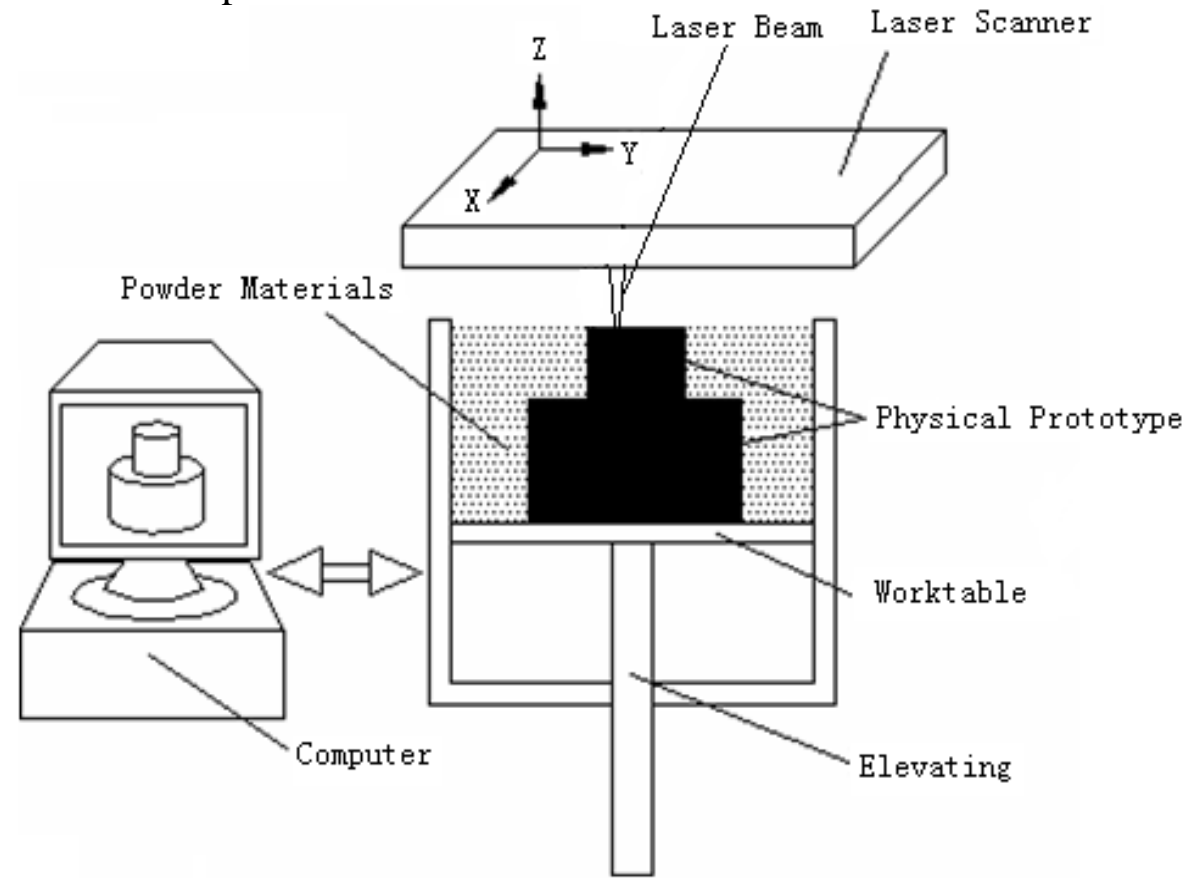

Fig.1 Process principle of 3D printing 
Step 4. Worktable falls down with the distance of one layer thickness and next layer of material is processed. Repeat the step 3 and step 4 until the physical prototype of part which is built by CAD system is obtained.

Step 5. The physical prototype is post-processed according to the product requirement.

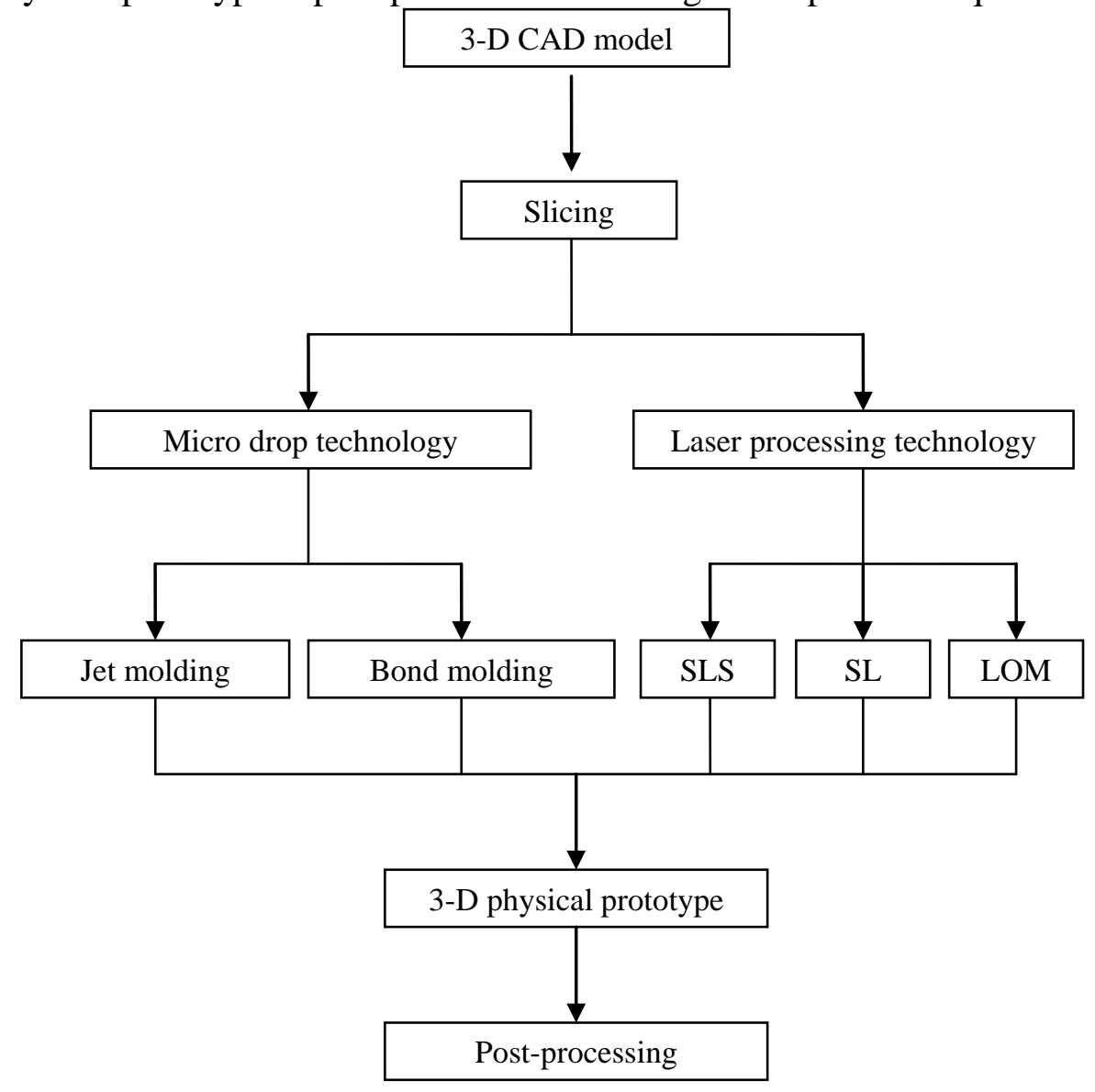

Fig.2 Processing flow chart of 3D printing

The process of 2D section scanning is the core process of 3D printing. The efficiency of this process determines the efficiency of whole 3D printing process. The 2D cross section data extracted by slicing the 3D CAD model is fragmented. So, if the data is transformed into processing path code directly, there will be a lot of idle running and the processing efficiency will be reduced. In response to this circumstance, a path optimization algorithm is developed in this paper.

\section{Path optimization algorithm}

The 2D section sliced from 3D CAD model is composed of one or more loops. There will be a lot of unnecessary idle running while the original data is output to transform the path scanning code, as shown in Fig.3. The solid line is 4 loops waiting to be processed, and the dashed line is idle running. The idle running is unnecessarily long.

The every single loop is a closed-path, so, the starting point of scanning is also the ending point. To avoid unnecessary idle running, the starting point and ending point of every single loop are reset. All the other points on the every single loop are re-sorted. By this algorithm, the starting point of currently processing loop is nearest the ending point of previous loop. The algorithm is described below.

Step 1. Resave the point sets data of every loop on the current 2D section. The point set coordinate of one loop saves as one array, and $F$ is the set include all array.

Step 2. The point $P$ in set $F$ nearest the origin of coordinates is the processing starting point and the loop which contains the point $P$ is called loop $P L$.

Step 3. Resort the points in loop $P L$ and save it in new set $N$. Delete the loop $P L$ in set $F$. 


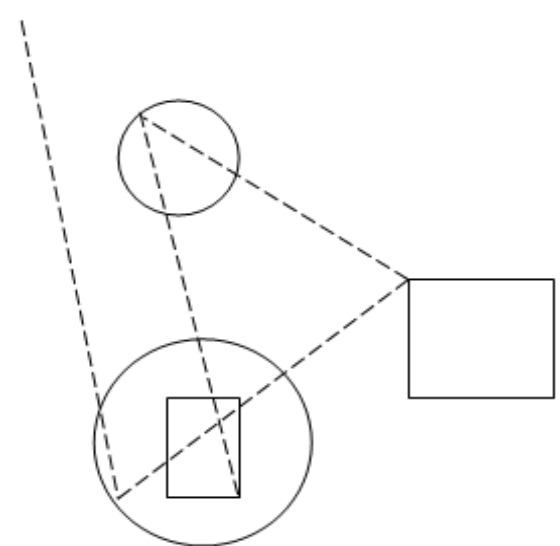

Fig.3 Un-optimized original path

Step 4. Find out the point $Q$ which is nearest point $P$ in set $F$. The loop which contains the point $Q$ is called loop $Q L$. Set $Q$ as the scanning starting point of loop $Q L$, also, as the end point of loop $Q L$.

Step 5. Resort the points in loop $Q L$ and save it in new set $N$. Delete the loop $Q L$ in set $F$.

Step 6. Repeat the step 4 and step 5, until $F=\Phi$. Then, all loops in set $N$ are the optimized paths in current 2D section.

Step 7. Regenerate the new scanning path by using the point data in set $N$.

Fig. 4 shows the Optimized path, and the idle running is shortest.

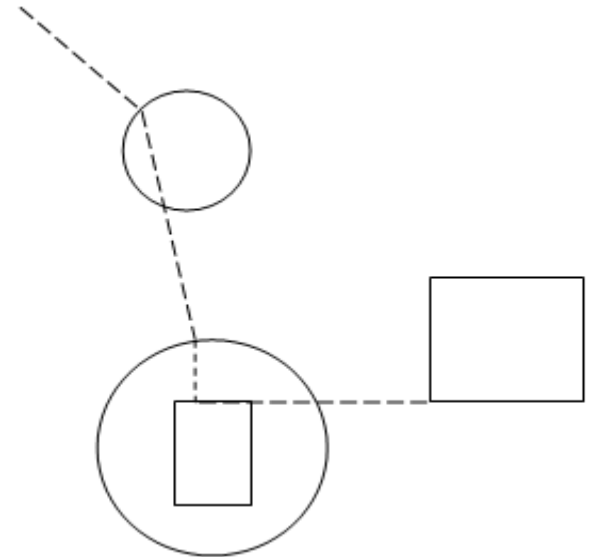

Fig.4 Optimized path

\section{Summary}

The process of 2D section scanning is the core process of 3D printing. If transform the original 2D section data into processing path code directly, there will be a lot of unnecessary idle running and the processing efficiency will be reduced. A path optimization algorithm using the minimum spanning tree strategy is developed in this paper. By this algorithm, the idle running is made the shortest. The case study results show that the efficiency of whole 3D printing is increased.

\section{Acknowledgement}

This work was supported by Natural Science Foundation of Guangdong Province \#2014A030307038

\section{References}

[1] J. P. Kruth, X. Wang, T. Laoui. Progress in Selective Laser Sintering. Annals of the CIPP, Vol. 42, (2001), No. 3, P. 21-38

[2] A. Wurikaixi, Z. W. Hua, Development of deformation measurement system in direct metal rapid prototyping. Computer Engineering and Applications, Vol. 25, (2008), No. 44, P. 246- 248 
[3] H. Y. Min, L. H. Yao. CAD/CAE/CAM intergration for increasing the accuracy of mask rapid prototyping system. Computers in Industry, Vol. 56, (2005), P. 442-456

[4] S. H. Choi, S. Samavedam. Modelling and optimisation of Rapid Prototyping. Computers in Industry, Vol. 47, (2002), P.39-53

[5] Y. J. Qiu, X. H. Zhou, X. P. Qian. Direct slicing of cloud data with guaranteed topology for rapid prototyping. The International Journal of Advanced Manufacturing Technology, Vol. 53, (2011), No. 4, P. 255-265

[6] K. S. Lee, S. H. Kim. Non-uniform deformation of an STL model satisfying error criteria. Computer-Aided Design, Vol. 42, (2010), No. 3, P. 238-247

[7] W. J. Ya, F. Liang, H. J. Bin, Adaptive slicing algorithm of STL model based on feature facets. Application Research of Computers, Vol. 28, (2011), No. 6, P. 2361-2364

[8] B. Starly, A. Lau, W. Sun. Direct slicing of STEP based NURBS models for layered manufacturing. Computer-Aided Design, Vol. 37, (2005), No. 4, P. 387-397

[9] H. Y. Min, L. H. Yao. Path planning effect for accuracy of rapid prototyping system. International journal of advanced manufacture and technology, (2006), No. 30, P. 233-246

[10] L. Luliano, M. Violante, A. Gatto, E. Bassoli, 3D printing technique applied to rapid casting. Rapid Prototyping Journal, Vol. 13, (2007), No. 3, P. 148-155 